\title{
BLOODSTAIN PATTERNS AND THE USE OF DIGITAL AID IN INTERPRETATION: A REVIEW
}

\author{
Triveni K \\ Department of Forensic Science \\ Jain deemed to be University, Bengaluru \\ Karnataka, India
}

\begin{abstract}
The study or examination of bloodstain patterns at the crime scene can be helpful in sequencing the events occurred and reconstructing the crime scene. The blood pattern analysis can determine the primary idea of type of crime that has occurred. Several people die as the reason of Homicide and blood patterns is found at such crime scenes. The various fields like physics, mathematics, and fluid mechanics is involved from documenting to interpreting the blood patterns. The bloodstain evidence at the crime scenes also contribute in testing DNA. The aim of this paper is to review the detailed information on types of crime scenes and the blood patterns that can be interpreted at the different crime scenes based on the previous studies and research. The technologies and developments to examine the crime scene with blood patterns is also studied with the help of research papers. The digital aid in particular is helpful in interpreting the bloodstain patterns.
\end{abstract}

Keywords - Crime scene, Bloodstains, Bloodstain pattern analysis, Blood spatter, Forensic Science

\section{INTRODUCTION}

Enormous spillage of blood is witnessed in most of the crime scenes. According to UCR program by FBI the crimes committed by blunt objects are more compared to rifle. Bloodstain pattern analysis at crime scenes will help the analyst and Investigation officer to recreate the actions between victim and perpetrator that caused the bloodshed. The evidence at crime scenes is studied based on Locard's exchange principle. It states that "whenever two objects are in contact, there will always be a mutual exchange of matter between them" (T.MillerEd.D., 2018). The bloodstain will be formed in different patterns depending on the crime, object used and surface on which blood in landing.

\section{BLOODSTAIN PATTERN ANALYSIS}

Bloodstain Pattern Analysis is a field in Forensic Science which is used in the reconstruction of the crime scene. The bloodstain pattern analysis takes place when violent crimes like murder, stabbing, crimes involving gunshot occurs. In such cases the BPA is done in order to understand the origin of blood. It is defined as "the scientific study of the static consequences resulting from dynamic blood shedding events" (Nabanita Basu, 2018). The analyst look at the patterns formed by bloodshed and tries to determine what happened at the crime scene. The evidence like fingerprints, tool marks or impressions can help in reconstruction of the logical sequence of events. The documenting and handling of bloodstains are important as they can't be taken to lab.

\section{A. Interaction of blood with the surfaces -}

The texture of target surface can change the shape and size of the bloodstain. The blood falling on smooth surface will have no distortion, whereas on rough surface the edges of blood drop will be distorted forming spines. Also blood drop landing in absorbent surface spreads due to capillary action and on non-absorbent surface it will repel forming thin parent stain (P. L. Kirk, 1974). The volume of blood, fall height, porosity and texture significantly affect the bloodstain pattern formed on fabrics (Baandyopadhyay \& Basu, 2015). Analysis research has been performed on fabrics, a study conducted by (Stephen Michielsen, 2015) showed the effect of textile surface on bloodstain and also has given demonstration on fabric interaction, distortion and alteration of stain. The experiment involving fluid dynamics that is imbibition effect of blood on fabrics along with direction of angles has been studied (Prashant Agrawal, 2017). There are works done on blood impact on surface and these studies are being very little help in verifying and interpreting the bloodstain. The condition of surface will also influence the final stain. If the surface is heated blood could impinge to surface. In arson cases the effects of extreme heat is seen where the heat is applied after blood is spilled which leaves bloodstain covered in soot (Larkin, 2015). The work involving blood on heat surface are yet to be studied in detail.

\section{B. Crime Scene involving bloodstain patterns -}

The bloodshed is caused when perpetrator commits violent crimes. Violent behaviour is involved in crimes like Homicide, assault, suicide and vehicular accidents. Investigators interpret bloodstain patterns to reconstruct the 


\section{International Journal of Engineering Applied Sciences and Technology, 2020 Vol. 5, Issue 4, ISSN No. 2455-2143, Pages 339-342 \\ Published Online August 2020 in IJEAST (http://www.ijeast.com)}

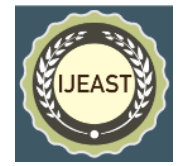

sequence of events that caused particular patterns (Franjic, 2019).

The sharp weapons like knife are used by culprit to stab a victim. Linear patterns of stains are formed in cases of stabbing, when the weapon is swung the blood flings onto nearby surface forming cast off spatter (A Simplified Guide to Forensic Science, 2013). The velocity of weapon on the target is medium in such cases. Swipe and wipe patterns are also seen if victim as suffered and fought back the perpetrator $(\mathrm{Li}$, 2015). The interpretation of position of victim, type of weapon found at scene and movement of victim after attack can help in investigation (Franjic, Blood in Murder Investigation, 2019).

The usages of guns to commit murders are recorded often. The gunshot injuries at scene forms mist like spatter (A Simplified Guide To Bloodstain Pattern Analysis). The entry wound shows the back spatter and exit wound forms forward spatter, the velocity of bullet is high on target and spatter depends on calibre of gun (A Forensic Guide for Crime Investigators, 2019). The occurrence of gunshot back spatter patterns by rifle and handgun was studied considering the parameters of bullet shape, size, speed and distance between blood source and target sheet (Daniel Attinger Y. L., 2018). The availability of bloodstain spatter pattern on hands in suicide cases involving firearms was explained with illustration of five cases (Kathrin Yen, 2003).

The brutal crime using blunt weapons like bat or hammer for hitting the victim forms impact spatter where larger area will collect more blood showing varying sizes of blood drops (A Simplified Guide To Bloodstain Pattern Analysis). The hammer is easily available to use as murder weapon and the print, stain patterns by hammer is analysed (Bandyopadhyay $\&$ Basu, 2015). Fresh swine blood was used to conduct experiment to show impact spatter formed by beating (Daniel Attinger Y. L., 2018).

The impact of object on target creates patterns, these patterns vary by the force target has received. The experiment by (Theresa Stotesbury, 2015) provides the understanding of spatter patterns formed from different velocities. The differentiation of impact spatter and cast off blood patterns are made possible by automated bloodstain pattern recognition system developed recently (R.M. Arthura, 2018).

\section{Interpretation of Bloodstain Patterns -}

While examining the bloodstains, stains can be latent or patent. The latent bloodstains are observed through chemiluminescent reaction when exposed to luminol reagent (Sumiko Polaccoa, 2019). The recording and photographic documentation of bloodstains are necessary steps prior to handling. The photographs are detailed using cameras with single lens reflective (SLR Camera) or digital single lens reflective (DSLR Camera) (Trumbo, 2011). The permanent markers are used to assist orientation of stains and to indicate directionality of stains in photographs (Duncan, 2007). The analysis of identification and aging of bloodstains is equally essential when it comes to bloodstain pattern analysis and it can be achieved by using chemo metric models (Vishal Sharma, 2018).

The determination of origin of blood landed on target from source is most essential in the investigation. The stain selection process for the analysis is important to estimate area of origin accurately. The theoretical model method has been used by bloodstain analyst on how to select stains. The recent study on using application method for the zone stain selection is proven that there is no significant difference from other methods (Stotesbury, 2015). The Stringing method has been a traditional method used by bloodstain pattern analyst to determine origin. The researchers have worked on this to introduce new methods which can provide more accuracy. The study was conducted for estimation of origin of blood by using the directional analysis method by focusing on the trimmed mean and a deterministic version of the RANSAC algorithm (M. Illes, 2013). The use of trigonometry in bloodstain pattern has been the standard method and globally used by analyst. Based on presumptions of value of distance, angle of impact of bloodstain and ability of blood to reach certain height and the angle of its impact, experiment was performed and the measurement of length of trajectory of impact and the height of blood sprayed upward from distance were compared and it was recommended to use trigonometry in bloodstain pattern analysis during an investigation (Peter Makovicky, 2013). Along with the trajectory analysis, the analyst depends on mathematical and statistical analysis to determine the likelihood of crime act and for more precision in analysis (MARTIN MATISOFF, 2012). The bloodstain shape model was used to calculate the impact angles which can help in estimation of area of origin (Joris, et al., 2014).

The digital aid in interpretation and analysis of bloodstain patterns has increased over a decade. The various computer programs are developed to analyse and produce accuracy in analysis. The calculation of blood source location from bloodstain pattern of downward moving drops was possible by the computer program called BackTrack TM (Illes, Carter, Laturnus, \& Yamashita, 2005). The 3D methods like string method or tangents method have proven to be useful in bloodstain pattern analysis. The 3D documentation method is made easily accessible and photogrammetric analysis for data interpretation can be done by little knowledge and experience (Ursula Buck, 2010). The software called Hemo vision has been developed and this was used to demonstrate and analyse the synthetic crime scene to check its approach (Philip Joris, 2015) In 2019, the program called Sherlock - web- based software was developed, it can estimate area of origin that does trajectory analysis using tangent method (Amanda Orr, 


\section{International Journal of Engineering Applied Sciences and Technology, 2020 \\ Vol. 5, Issue 4, ISSN No. 2455-2143, Pages 339-342 \\ Published Online August 2020 in IJEAST (http://www.ijeast.com)}

2019). The most recent study was on 3D scanning, it was aimed to see if FARO Focus 3D Scanner and FARO Zone 3D software were to be more accurate compared to manual method (Owen Esaias, 2020).

\section{CONCLUSION}

The bloodstain patterns made by the bloodshed event are different depending on the type of crime. The most crimes are caused by blunt forces which leaves the certain patterns on the surfaces at crime scene. The sequence of events and position of perpetrator is crucial in a crime scene. The neat documentation of crime scene is the primary step of the analysis. Tools and computer programs have tremendously aided in process of bloodstain pattern analysis. There are methodologies developed for directional analysis and location of the area of origin of the impact spatter in crime scene. The research work is being done to develop many programs which can provide more accuracy and precision in interpretation of bloodstain patterns. The age old Biblical dictum "Blood never lies" is the base for the research on Bloodstain Pattern Analysis. This review concludes that developing more computerised programs and tools can aid in better understanding, more precise analysis of interpretation of bloodstain patterns.

\section{REFERENCE}

[1] T.MillerEd.D., A. 1. (2018). A Practical Exercise of the Locard Exchange Principle. Crime Scene Investigation Laboratory Manual (Second Edition), 13-17.

[2] Nabanita Basu, S. K. (2018). Bloodstain Pattern Analysis and Reconstruction. International Journal of Trend in Research and Development , 5(3), 227-236.

[3] P. L. Kirk, J. I. (1974). Crime Investigation. In J. I. P. L. Kirk, Crime Investigation. John Wiley \& Sons Inc.

[4] Samir Kumar Bandyopadhyay, N. B. (2015). Bloodstains Analysis on Fabric from Contact in a Crime Scene. International Journal of Computer System , 2 (1), 30-32.

[5] Stephen Michielsen, M. T. (2015). Bloodstain Patterns on Textile Surfaces: A Fundamental Analysis. U.S. Department of Justice.

[6] Prashant Agrawal, L. B. (2017). Bloodstains on woven fabric: Simulations and experiments for quantifying the uncertainty on the impact and directional angles. Forensic Science International , 240-252.

[7] LARKIN, B. A. (2015). Bloodstain pattern analysis: scratching the surface. Manchester Metropolitan University.

[8] Franjić, S. (2019). Bloodstain Pattern Analysis Provides Evidence during Crime Scene Investigation. Instant Journal of Forensic Science, 33-38.

[9] National Forensic Science Technology Center. (n.d.).
[10] LI, R. (2015). Forensic Biology (Second Edition). In R. LI, Forensic Biology (Second Edition) (p. 46). CRC Press.

[11] Franjić, S. (2019). Blood in Murder Investigation. Journal of Law and Judicial System , 2 (3), 18-22.

[12] National Forensic Science Technology Center. (n.d.).

[13] NICFS. (n.d.).

[14] Daniel Attinger, Y. L. (2018). A data set of bloodstain patterns for teaching and research in bloodstain pattern analysis: Gunshot backspatters. Data in Brief, 269-278.

[15] Kathrin Yen, M. M. (2003). Blood-Spatter Patterns. The American Journal of Forensic Medicine and Pathology , 24, 132-140.

[16] National Forensic Science Technology Center. (n.d.).

[17] Samir Kumar Bandyopadhyay, N. B. (2015). Blood Stain with Hammer Imprint Shown in Crime Scene. Open Access Library Journal .

[18] Daniel Attinger, Y. L. (2018). A data set of bloodstain patterns for teaching and research in bloodstain pattern analysis: Impact beating spatters. Data in Brief , 648-654.

[19] Theresa Stotesbury, M. I. (2015). An Impact Velocity Device Design for Blood Spatter Pattern Generation with Considerations for High-Speed Video Analysis. Journal of Forensic Science, 1-8.

[20] R.M. Arthura, J. H. (2018). An automated approach to the classification of impact spatter and cast-off bloodstain patterns . Forensic Science International , 310-319.

[21] Sumiko Polaccoa, P. W. (2019). Luminol reagent control materials in bloodstain pattern analysis: A silicon sol-gel polymer alternative . Forensic Chemistry , 91-98.

[22] Trumbo, W. Y. (2011). Scientific approaches in bloodstain analysis (crime scene investigation) . National university.

[23] Duncan, C. (2007). Bloodstain Photography. 4-13.

[24] Vishal Sharma, R. K. (2018). Trends of Chemometrics in Bloodstain Investigations. Trends in Analytical Chemistry , 181-195.

[25] Stotesbury, M. I. (2015). Development of an application method for a zone stain selection model in bloodstain pattern analysis . Canadian Society of Forensic Science Journal , 1-7.

[26] M. Illes, M. B. (2013). Robust estimation for area of origin in bloodstain pattern analysis via directional analysis. Forensic Science International , 223-229.

[27] Peter Makovicky, P. H. (2013). The use of trigonometry in bloodstain analysis. Soudní lékarství / casopis Sekce soudního lékarstvi Cs. lékarské spolecnosti J. Ev. Purkyne , 20-25.

[28] MARTIN MATISOFF, L. B. (2012). Mathematical \& Statistical Analysis of Bloodstain Pattern Evidence. CE ARTICLE , 81. 
[29] Philip Joris, W. D. (2014). Calculation of bloodstain impact angles using an Active Bloodstain Shape Model. Journal of Forensic Radiology and Imaging , 188-198.

[30] M.B. ILLES, A. C. (2005). Use of the BackTrack TM Computer Program for Bloodstain Pattern Analysis of Stains from Downward-Moving Drops. Journal of the Canadian Society of Forensic Science, 213-218.

[31] Ursula Buck, B. K. (2010). 3D bloodstain pattern analysis: Ballistic reconstruction of the trajectories of blood drops and determination of the centres of origin of the bloodstains. Forensic Science International , 22-28.

[32] Philip Joris, W. D. (2015). HemoVision: An automated and virtual approach to bloodstain pattern analysis. Forensic Science International , 116-123.

[33] Amanda Orr, M. I. (2019). Validation of Sherlock, a linear trajectory analysis program for use in bloodstain pattern analysis. Canadian Society of Forensic Science Journal , 1-17.

[34] Owen Esaias, G. N. (2020). Improved area of origin estimation for bloodstain pattern analysis using 3D scanning. Journal of Forensic Science , 65, 722-728. 